\title{
A Histological and an Immunohistochemical Study on the Effects of Iron Overdose on the Basal Ganglia of the Adult Albino Rat
}

\author{
Original \\ Mohamed Nabil Mahmoud Saleh, Mohamed El-Badry Mohamed, Ayman S. Amer, \\ Article \\ Omnia I. Ismail
}

Human Anatomy and Embryology Department, Faculty of Medicine, Assiut University, Assiut, Egypt.

\begin{abstract}
Background: Iron is the most abundant element on earth and an essential metal for life. It is used extensively by proteins involved in the electron transport chain, the active centers of many enzymes and oxygen transport. It is essential for the adequate development and functioning of the brain. The regulation of the iron metabolism is crucial since both the iron deficiency and the iron overload can cause a disease.

Aim of the Work: To detect the effects of iron exposure during the postnatal period on the putamen, the subthalamic nucleus and the substantia nigra in adult albino rats.

Material and Methods: A total number of twenty albino rats were used in the study. They were equally divided into a control group and an experimental group. The control group received tap water orally. The experimental group received $15 \mathrm{mg} /$ $\mathrm{kg}$ of ferrous gluconate orally. The regimen started at postnatal day 12 and continued until three months old. The rats were anaesthetized and the brains were extracted. The specimens from the fixed brains were dissected and processed for the light and the electron microscopic examination. Morphometric measurements were also done.

Results: The light microscopic study of the treated group revealed neurons of putamen had dense darkly stained nuclei and vacuolations appeared within the neuropil. Wide spaces between darkly stained neurons of the subthalamic nucleus were detected. The neuropil of the substantia nigra pars compacta ( $\mathrm{SNc}$ ) had many vacuoles and most of the neurons had darkly stained nuclei. Immunohistochemistry of the putamen using anti-TH demonstrated a reduction of TH expression in a patchy manner. Immunohistochemistry of SNc showed a weak TH immunoreactivity in the neuropil of the treated group and a reduction in the number of $\mathrm{TH}$ immunopositive neurons in comparison with the control group. The electron microscopic study of the SNc and putamen of the treated group showed degeneration of the mitochondria, vacuolization of the cytoplasm, heterochromatic nuclei with irregular outline and marked loss of cell organelles in the cytoplasm. Morphometric studies revealed significant reduction in the cell count and surface area of the neurons in SNc and putamen of the treated group in comparison with the control group.

Conclusion: Iron overdose during postnatal period produces degeneration of the putamen, subthalamic nucleus and substantia nigra in the adult albino rat.
\end{abstract}

Received: 26 March 2018, Accepted: 03 September 2018

Key Words: Albino rat, iron, putamen, Substania nigra, Subthalamic nucleus.

Corresponding Author: Omnia I. Ismail, Human Anatomy and Embryology Department, Faculty of Medicine, Assiut University, Assiut, Egypt, Tel.: +20 1002070846, Fax:+20 88 2343703, E-mail: omnia8383@yahoo.com.

ISSN: 1110-0559, Vol. 41, No. 4

\section{INTRODUCTION}

Iron is an important element for normal cellular functions, and formation of hemoglobin, which transports oxygen. It is a key component of cytochromes and the ironsulfur complexes of the oxidative chain and is important for producing adenosine triphosphate (ATP). Iron participates in a wide spectrum of cellular functions ${ }^{[1]}$. Additional demands for iron in the brain come from myelogenesis and myelin maintenance ${ }^{[2]}$. Oligodendrocytes, the cells responsible for myelin production and maintenance in the CNS are enriched for iron relative to other cells in the brain $^{[3]}$. Normal exposure to iron occurs through the diet and iron supplementation. Iron exposure occurs through occupational exposures, mainly from metal fumes or metal dust, as would be generated during welding and in iron and steel industry ${ }^{[4]}$.

Iron enters the brain through the blood-brain barrier and the choroid plexus-cerebrospinal fluid barrier ${ }^{[5]}$. Iron storage in the neurons of the SN occurs through binding of iron within neuromelanin ${ }^{[6]}$. Neuromelanin is the oxidation product of dopamine and tyrosinase is the enzyme used for melanin synthesis in the body ${ }^{[7]}$.

The accumulation of iron in the substantia nigra pars compacta is the key factor in pathogenisis of $\mathrm{PD}^{[8]}$.

Although iron is not present at birth, progressive iron deposition occurs in different structures of the brain during aging process and the extent of iron deposition with aging 
is markedly higher in the basal ganglia than in most of the other brain structures ${ }^{[9-10]}$. Increased brain iron levels may be a risk factor for age-related neurologic disorders, such as Alzheimer's disease ${ }^{[1]}$.

Iron supplementation in a critical neonatal period induced oxidative stress in the adult life in selective brain regions as substantia nigra and striatum ${ }^{[11]}$.

\section{MATERIALS AND METHODS}

\section{Chemicals}

Ferrous gluconate powder $(\mathrm{Fe} 2+)$ was purchased from CID, Assuit, Egypt. Anti tyrosine hydroxylase was purchased from Sigma (St. Louis, MO, USA).Secondary antibody (antipolyclonal universal kit) was purchased from Sigma (St. Louis, MO, USA).

\section{Animals}

A total number of 20 twelve days old albino rats were used in this study. Each rat weight was about 35 gm. The animals were obtained from the Animal House, Assiut University. The experiment was approved by the Institutional Ethics Committee of Assiut University.

\section{Experimental design}

The rats were settled in well- ventilated stainless steel cages. They were separated randomly into control and experimental groups (each consisted of 10 rats). The experimental group received daily $15 \mathrm{mg} / \mathrm{kg}$ of Ferrous gluconate $(\mathrm{Fe} 2+)$ dissolved in distilled water orally through a gastric tube from postnatal days 12 till three month. The used dose in this work was according to ${ }^{[11,12]}$. The control group was given $0.5 \mathrm{ml}$ distilled water daily via the same route and for the same period.

At the time of scarification, the two groups were anaesthetized by ether inhalation, subjected to an intracardiac perfusion by normal saline $0.9 \% \mathrm{NaCl}$ then sacrificed. Brain specimens were extracted from the two groups.

\section{Light microscopic study:}

The brain specimens were fixed in $10 \%$ neutral buffered formalin, PH 7.4 then processed for paraffin blocks. The brain specimens were subjected for the Nissl's stain (Einersons gallocyanine) according to ${ }^{[13]}$. Anti Tyrosine hydroxylase (TH) immunohistochemical staining used as a marker for dopamine-producing neurons (rabbit anti-TH, 1:10,000; Gene Tex 113016). It was diluted in the phosphate buffered saline. For positive control staining, paraffin sections of the rat adrenal gland were immunostained by the same procedure and indicated positive immunoreactivity for $\mathrm{TH}$ in the adrenal medulla. For negative control staining, sections were processed in an identical manner but incubated with PBS instead of the primary antibody and these sections failed to show immunolabeling for $\mathrm{TH}^{[14]}$. Slides were incubated with anti-TH overnight at the $4{ }^{\circ} \mathrm{c}$, then washed several times. Secondary antibody was then applied for 2 hours at room temperature. The reaction was indicated using diaminobenzidine, positivity was seen as a brown color.

\section{Electron microscopic study:}

The animals were perfused intracardially by saline then by $2.5 \%$ gluteraldehyde in the sodium cacodylate buffer at pH 1.5 for the preparation of semithin sections. The brains were cut into two halves by a sagittal section and kept in the same fixative for an average period of 24 hours. The coronal section was taken at the level of the mamillary body and a sample of the putamen was taken and from the midbrain a second sample of the substantia nigra was taken. Post fixative was added in 1\% Osmium Tetroxide for 1 hour. The fixative was washed out in distilled water 3 times in 10 minutes changes. Dehydration series were done as follows: $30 \%$ ethanol for 10 minutes, $50 \%$ ethanol for 10 minutes, $70 \%$ ethanol for 10 minutes, $90 \%$ ethanol for 10 minutes and $100 \%$ ethanol for 10 minutes, respectively ${ }^{[15]}$.

The samples were embedded in fresh resin and left overnight at $60 \mathrm{oC}$. Sectioning was done to produce semithin slices of specimen. They were cut by an ultramicrotome with a diamond knife to produce ultra-thin sections of 60 $90 \mathrm{~nm}$ thick. The sections were stained for several minutes by double staining technique, with an aqueous or alcoholic solution of uranyl acetate followed by aqueous lead citrate $^{[15]}$. The sections were examined and photographed by the transmission electron microscope (TEM) ("Jeol" E.M.-100 CX11; Japan) at the Electron Microscopic Unit of Assiut University.

\section{Morphometric study:}

The semithin sections were studied morphometrically for the following parameters in both control and treated groups sections using computerized image analyzer system software (Leica Q 500 MCO; Leica, Wetzlar, Germany) attached to a Leica universal microscope with a camera at the Histology Department, Faculty of Medicine, Assiut University, Egypt:

1. Number of the neurons in the putamen and substantia nigra.

2. Surface area of the neuronal perikarya in the putamen and the substantia nigra.

\section{Statistical analysis:}

The above parameters were calculated for 6 animals in each group studied. The mean value and standard deviation were calculated for each parameter. The SPSS program was used and unpaired student's t-test was applied to compare between the two groups ${ }^{[16]}$.

\section{RESULTS}

- The control group:

\section{The putamen}

\section{1- Light microscopic study:}

The putamen stained with gallocyanine displayed the 
normal histological structure. The neurons had vesicular nuclei, and basophilic cytoplasm. The neurons were surrounded by perineuronal spaces. The striatal fibers, glial cells and neuropil were noticed (Fig. 1).

\section{2-Immunohistochemical study:}

Immunohistochemistry of the putamen showed many positive TH-immunoreactive neurons and a positive TH immunoreactivity in the striatal fibers and neuropil (Fig. 2)

\section{3-Electron microscopic study:}

Semithin sections of the putamen revealed the neurons with rounded or oval nuclei with prominent nucleoli. The glial cells are small in the size than the neurons and showed dense nuclei (Fig. 1).

Ultrathin sections of the putamen showed neurons with euchromatic nucleus and prominent nucleolus. The cytoplasm showed rough endoplasmic reticulum, many free polyribosomes and scattered mitochondria (Fig. 3). The symmetrical synapses appeared with many vesicles and mitochondria (Fig. 4) and blood vessels are seen with normal endothelial cells and pericytic microglia (Fig. 5).

\section{The subthalamic nucleus}

Coronal sections of the brain stained with gallocyanine showed the biconvex lens shaped subthalamic nucleus which consisted of multiple packed dense neurons (Fig. 6).

\section{The substantia nigra pars compacta}

\section{1- Light microscopic study:}

The substantia nigra pars compacta $(\mathrm{SNc})$ was shown as a band of closely packed neurons that had variable sizes and shapes of neuronal perikarya. Their cytoplasm showed moderate to intense basophilia (Fig. 7).

\section{2- Immunohistochemical study:}

Immunohistochemistry of SNc showed many positive TH-immunoreactive neurons. TH-immunoreactive processes were seen within a dense $\mathrm{TH}$-immunoreactive neuropil (Fig. 8).

\section{3- Electron microscopic study:}

Semithin toluidine blue-stained sections showed a group of closely packed neuronal perikarya. Their large pale nuclei appeared nearly rounded. Their cytoplasm was granular basophilic. The glial cells were small in size than the neurons (Fig. 7).

Electron micrographs of the neurons showed a large euchromatic nucleus with a prominent nucleolus. The cytoplasm contained distinct aggregates of short RER cisternae with many free polyribosomes between them and scattered mitochondria (Fig. 9). The asymmetrical synapses with many synaptic vesicles and mitochondria were observed in the SNc (Fig. 10).

\section{- Treated group:}

\section{The putamen}

\section{1- Light microscopic study:}

Some neurons had dense darkly stained nuclei and other neurons showed vesicular nuclei. The neurons were surrounded by perineuronal spaces. The blood vessels appeared dilated. Some vacuolations appeared within the neuropil (Fig. 11).

\section{2- Immunohistochemical study:}

Immunohistochemistry of the putamen using anti- TH demonstrated some areas with a positive $\mathrm{TH}$ immunoreactivity with relative reduction in intensity of immunoreativity and other areas with a negative TH immunoreaction. Dilated blood vessels were noticed. Many vacuolations within the striatal fibers and neuropil were seen (Fig. 12).

\section{3- Electron microscopic study:}

Examination of semithin sections of the putamen showed neurons having darkly stained rounded nuclei. Other neurons had nuclei with condensation of the chromatin in the periphery. Other darken shrunken neurons were observed. The neuron showed irregular outline and vacuolation. Other shrunken neurons with pyknotic nuclei were seen. The condensed and shrunken striatal fibers and vacuolation were also observed (Fig. 11). Examination of an ultrathin section in the putamen showed neurons with heterochromatic nuclei. Indentations of the nuclear membrane were observed (Fig. 13). The cytoplasm appeared as electron dense. There were vacuolations and a marked loss of cell organelles within the cytoplasm. Some neurons had irregular nuclei with deep invaginations of the nuclear membranes. The cytoplasm showed a dilated rough endoplasmic reticulum, damaged mitochondria and some polyribosomes (Figs. 13). At the site of synapses, there were an area of loss of presynaptic and postsynaptic densities and the synaptic terminal showed minute number of the synaptic vesicles. Swollen mitochondria with destructed inner cristae were observed and their walls were broken at several points (Fig. 14). The endothelial cells of the blood vessels had dark flat nuclei with dense clumps of chromatin. The pericytic microglia showed big vacuolations, a shadow of lost nucleus, damaged mitochondria and rarified cytoplasm (Fig. 15)

\section{The subthalamic nucleus}

The subthalamic nucleus was stained with gallocyanine and showed wide spaces between darkly stained neurons (Fig. 16).

\section{The substantia nigra pars compacta}

\section{1- Light microscopic study:}

The substantia nigra pars compacta had varying sizes and shapes of the neuronal perikarya. The neuropil had many vacuoles. Most of the neurons appeared to have darkly stained nuclei (Fig. 17). 


\section{2- Immunohistochemical study:}

Immunohistochemistry of $\mathrm{SNc}$ showed weak $\mathrm{TH}$ immunoreactivity and reduction in the number of $\mathrm{TH}$ immunopositive neurons and mild immunoreactive neuropil (Fig. 18).

\section{3- Electron microscopic study:}

The semithin section in the substantia nigra pars compacta demonstrated apparently increased interstitial spaces between neurons and a decreased number of neuronal perikarya compared with control. Some neurons appeared normal with vesicular nuclei and prominent nucleoli. Other neurons had deeply stained nuclei with irregular outlines. Some neurons with pyknosis were seen.

In the ultrathin sections of the substantia nigra pars compacta, the neurons had heterochromatic nuclei with irregular outlines. The cytoplasm appeared to be rarified. Marked loss of cell organelles of the cytoplasm was observed (Fig. 19). Some shrunken pyknotic neurons were seen. The nucleus showed condensation of the chromatin in the periphery with irregular outline and points of breakage in the nuclear membrane. The cytoplasm showed few damaged mitochondria, dilated rough endoplasmic reticulum, vacuoles and few free polyribosomes (Fig. 19). Symmetrical synapses were observed in the SNc and showed an area of loss of presynaptic and postsynaptic densities with a few synaptic vesicles and destructed mitochondria with breakage of their inner cristae (Fig. 20).

\section{Morphometric study \\ Cell count of putamen}

The mean number of cells using $\times 400$ magnification in the putamen per an area of $313.4 \mu^{2}$ in the control group is

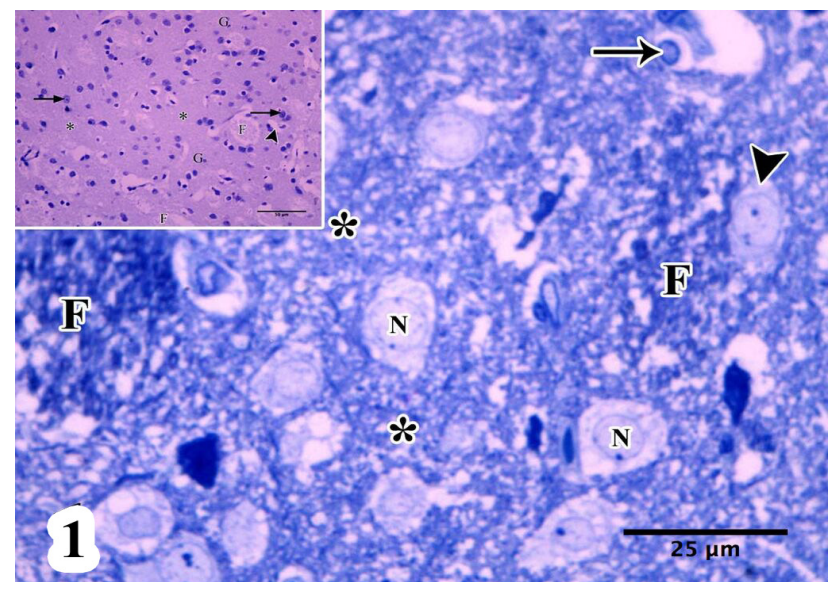

Fig. 1: A semithin section of the putamen of adult control rat showing the neurons having lightly stained rounded and oval nuclei $(N)$ surrounded by pale basophilic cytoplasm (arrowhead). The glial cells are smaller and darker than the neurons and have dense nuclei (arrow).The striatal fibers (F) and neuropil (asterisks) are seen. Inset: A photomicrograph of adult control rat putamen showing neurons (arrows) with vesicular nuclei and basophilic cytoplasm. The neurons were surrounded by perineuronal spaces (arrow head). The striatal fibers (F), glial cells (G) and neuropil (asterisk) are noticed. Toluidine blue, $\times 1000$ and Inset: Gallocyanine, $\times 400$ found to be $51.6 \pm 2.2$, while it is found to be $31.0 \pm 2.9$ in the treated group as shown in (Table 1 and Histogram 1). It appears that there is a decrease in the mean number of cells in the treated groups. This decrease in the mean number of cells is significant $(p<0.001)$.

\section{Surface area of neuron in the putamen}

The surface area of neurons using $\times 1000$ magnification in the putamen per an area of $124.2 \mu^{2}$ in the control group is found to be $100.4 \pm 2.5$, while it is found to be $64.2 \pm 4.9$ in the treated group as shown in (Table 2 and Histogram 2). It appears that there is a reduction in the surface area in the treated groups. This decrease is statistically significant $(p<0.001)$.

\section{Substantia nigra pars compacta}

\section{Cell count of the substantia nigra pars compacta}

The mean number of cells in SNc per an area of $313.4 \mu$ ${ }^{2}$ using $\times 400$ magnification in the control group is found to be $62.2 \pm 1.3$, while it is found to be $29.1 \pm 1.4$ in the treated group as shown in (Table 3 and Histogram 3). It appears that there is a reduction in the mean number of cells in the treated groups. This decrease in the mean number of cells is very highly significant $(p<0.001)$.

\section{Surface area of the neurons in substantia nigra pars compacta}

The surface area of neuron in SNc per an area of 124.2 $\mu^{2}$ using $\times 1000$ magnification in the control group is found to be $125.0 \pm 1.7$, while it is found to be $25.1 \pm 3.4$ in the treated group as shown in (Table 4 and Histogram 4). It appears that there is a reduction in the surface area in the treated groups. This decrease is statistically significant $(p<0.001)$.

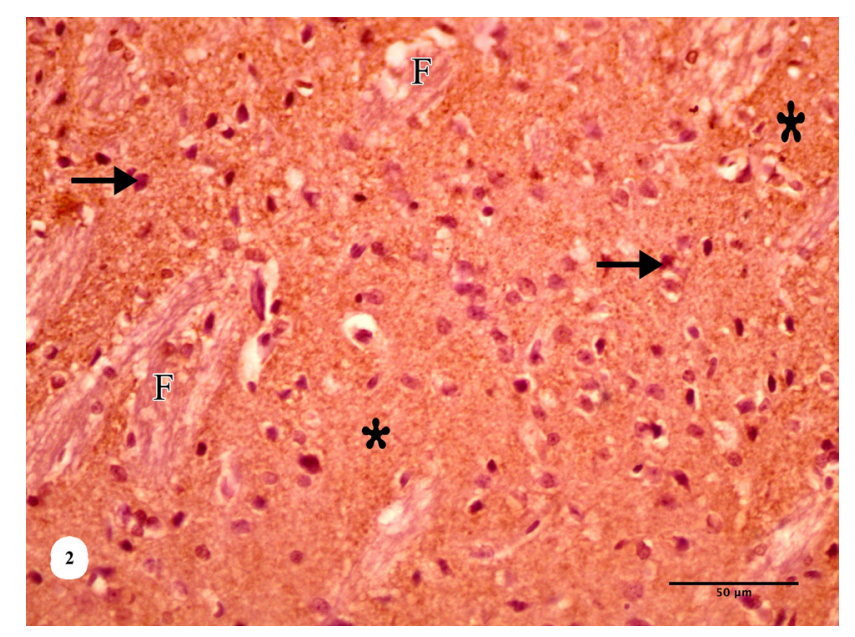

Fig. 2: A photomicrograph of adult control rat putamen showing a positive TH immunoreactivity in the neurons (arrows), striatal fibers (F) and neuropil (asterisks). TH, counterstained with $\mathrm{H}, \times 400$ 


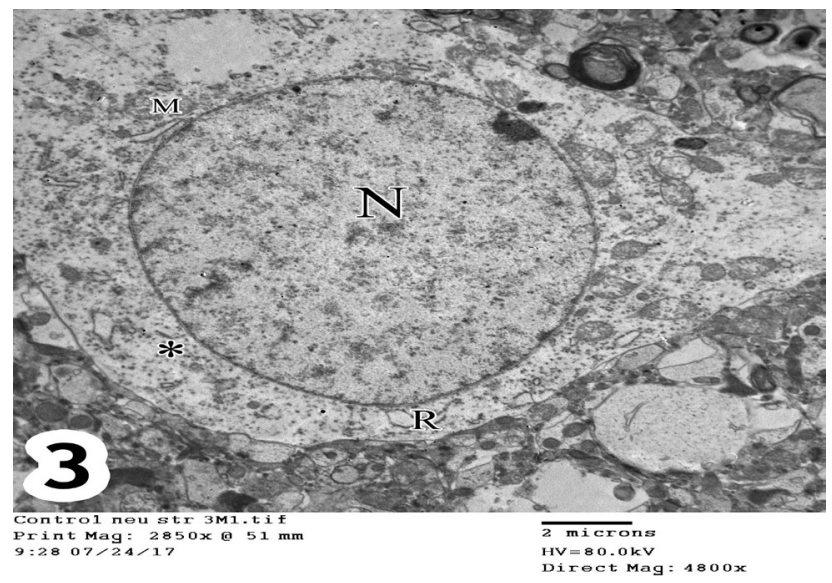

Fig. 3: An electron micrograph of an ultrathin section in the putamen of adult control rat showing a large neuron with an euchromatic nucleus $(\mathrm{N})$. The cytoplasm shows rough endoplasmic reticulum (R), polyribosomes (asterisk) and scattered mitochondria (M). TEM, $\times 4800$

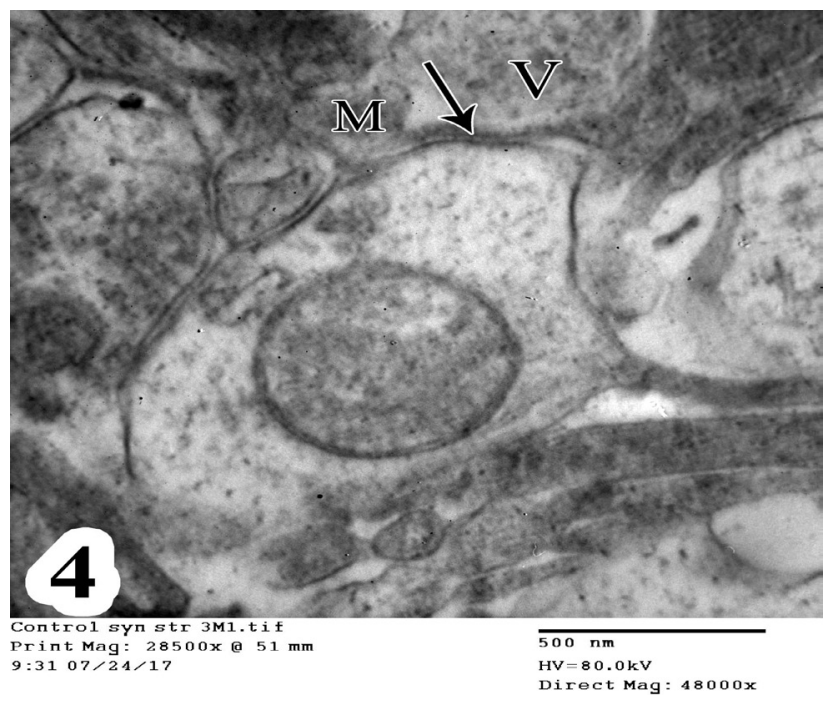

Fig. 4: An electron micrograph of an ultrathin section in the putamen of adult control rat showing symmetrical synapse (arrow) with many vesicles $(\mathrm{V})$ and mitochondria $(\mathrm{M})$. TEM, $\times 48000$

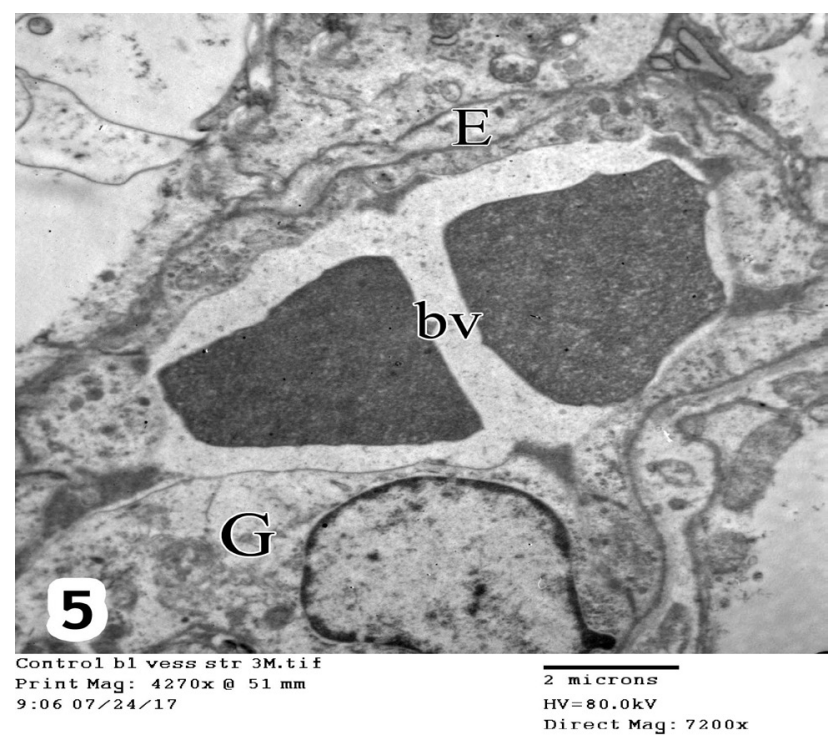

Fig. 5: An electron micrograph of an ultrathin section in the putamen of adult control rat showing a blood vessel (bv) with normal endothelial cells (E) and pericytic microglia (G).

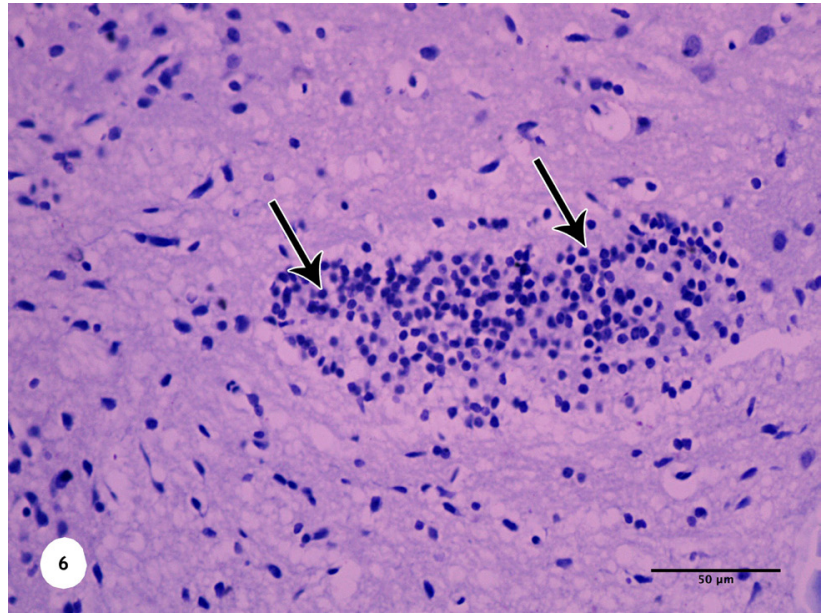

Fig. 6: A photomicrograph of the subthalamic nucleus of adult control rat showing multiple packed dense neurons (arrows) forming characteristic biconvex lens shaped subthalamic nucleus. Gallocyanine, $\times 400$

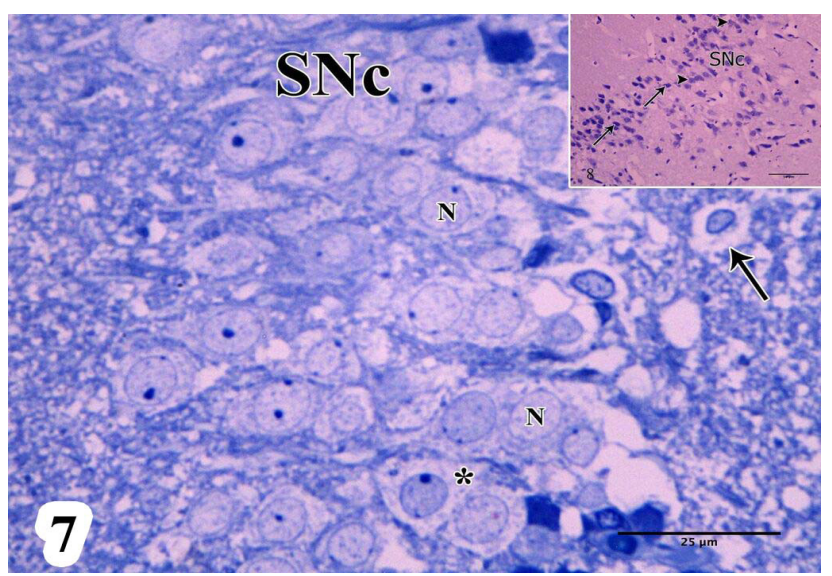

Fig. 7: A photomicrograph of a semithin section in the substantia nigra pars compacta $(\mathrm{SNc})$ of an adult control rat showing a group of closely packed neuronal perikarya. Their large pale nuclei $(\mathrm{N})$ appeared nearly rounded. Their cytoplasm appears granular basophilic (asterisk). The glial cells are smaller and darker than the neurons and have dense nuclei (arrow). Inset: A photomicrograph of adult control rat midbrain showing the substantia nigra pars compacta $(\mathrm{SNc})$ showing variable sizes and shapes of neuronal perikarya. Their cytoplasm shows moderate (arrowhead) to intense (arrow) basophilia. Toluidine blue, $\times 1000$ and Inset: Gallocyanine, $\times 400$

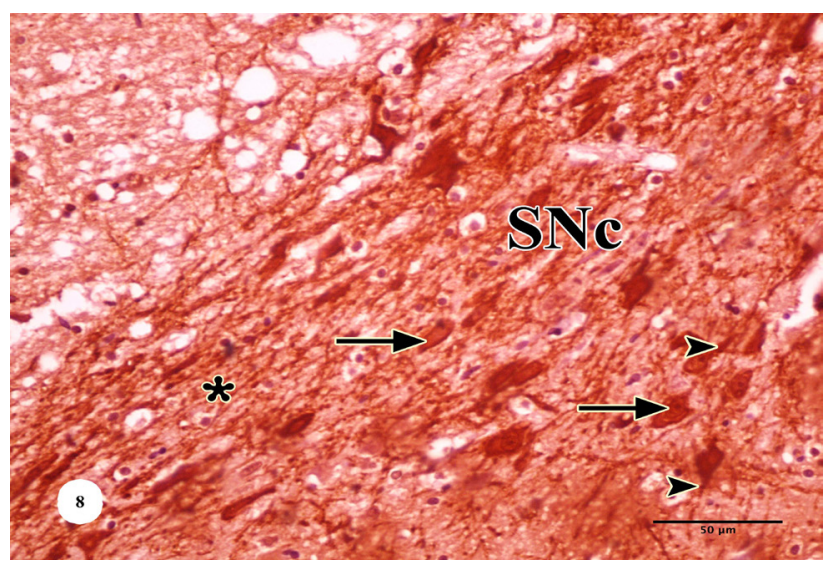

Fig. 8: A photomicrograph of adult control rat substantia nigra pars compacta ( $\mathrm{SNc}$ ) showing strong positive $\mathrm{TH}$ immunoreactive neurons (arrows) and their TH-immunoreactive processes (arrow head) that give rise to an intense $\mathrm{TH}$-immunoreactive neuropil (asterisk). $\mathrm{TH}$, counterstained with $\mathrm{H}, \times 400$ 


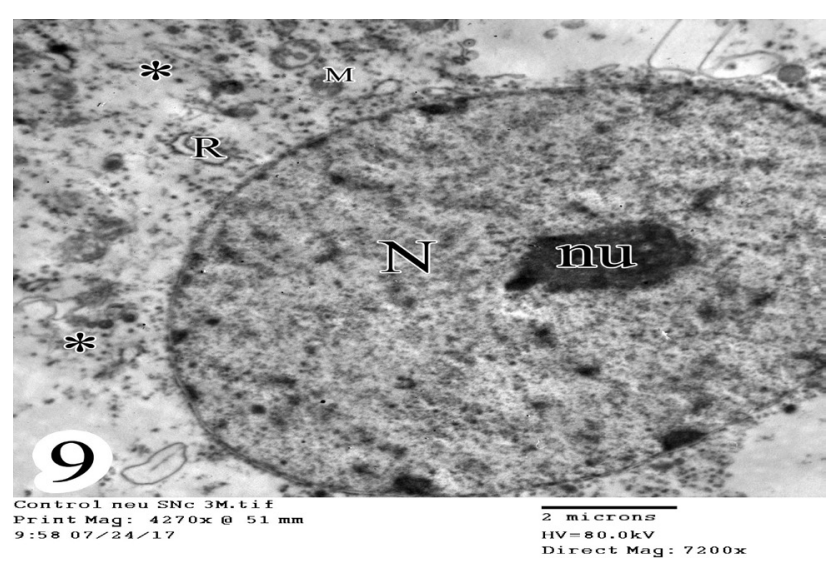

Fig. 9: An electron micrograph of an ultrathin section in the substantia nigra pars compacta of adult control rat showing a part of a large neuron with an euchromatic nucleus $(\mathrm{N})$, a prominent nucleolus $(\mathrm{nu})$. Aggregates of rough endoplasmic reticulum (R), many free polyribosomes (asterisks) and scattered mitochondria (M) are seen in the cytoplasm. TEM, $\times 7200$

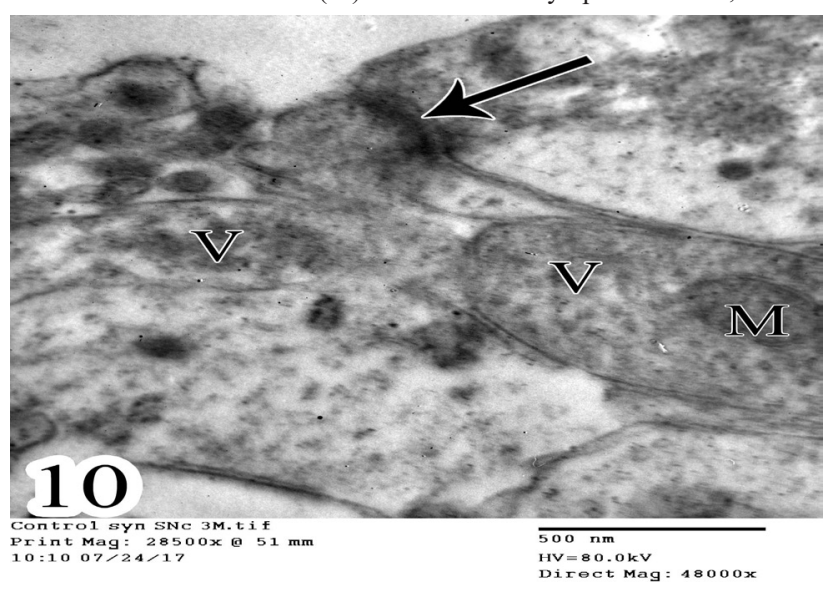

Fig. 10: Transmission micrograph of an ultrathin section in the substantia nigra pars compacta of adult control rat showing asymmetrical synapse (arrow) with many synaptic vesicles (V) and normal mitochondria (M).TEM, $\times 48000$

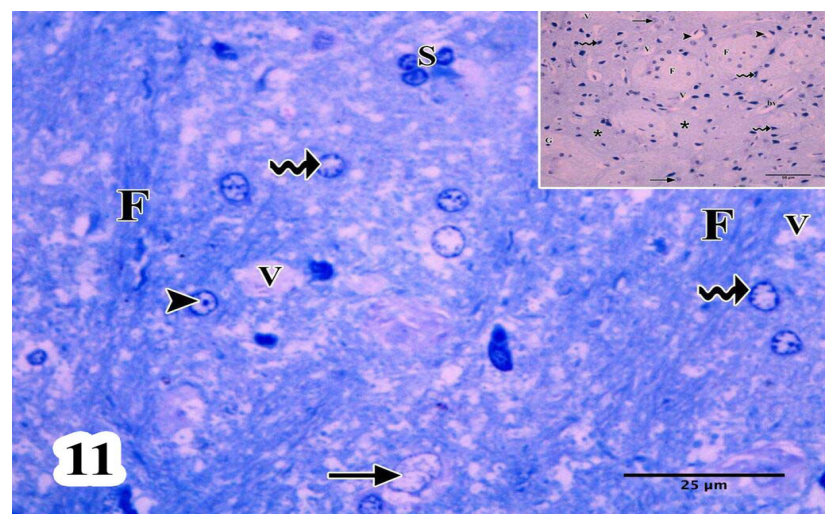

Fig. 11: A semithin section of the putamen of adult treated rat showing some neurons having rounded nuclei with nucleoli (arrow head).Other neurons (curved arrow) have nuclei with peripheral condensation of the chromatin. Other deeply stained shrunken neurons (S) are observed. The neuron (arrow) shows irregular outline and vacuolation. Condensed and shrunken striatal fibers (F) and vacuolations (V) are seen. Inset: A photomicrograph of the putamen of adult treated rat showing multiple neurons with darkly stained nuclei (curved arrows) and some neurons with vesicular nuclei (arrows). The neurons are surrounded by perineuronal spaces (arrow head). The blood vessels (bv) appear dilated. The striatal fibers (F) and neuropil (asterisk) are noticed. Some vacuolations appear within neuropil (V). Toluidine blue, $\times 1000$ and Inset: Gallocyanine, $\times 400$

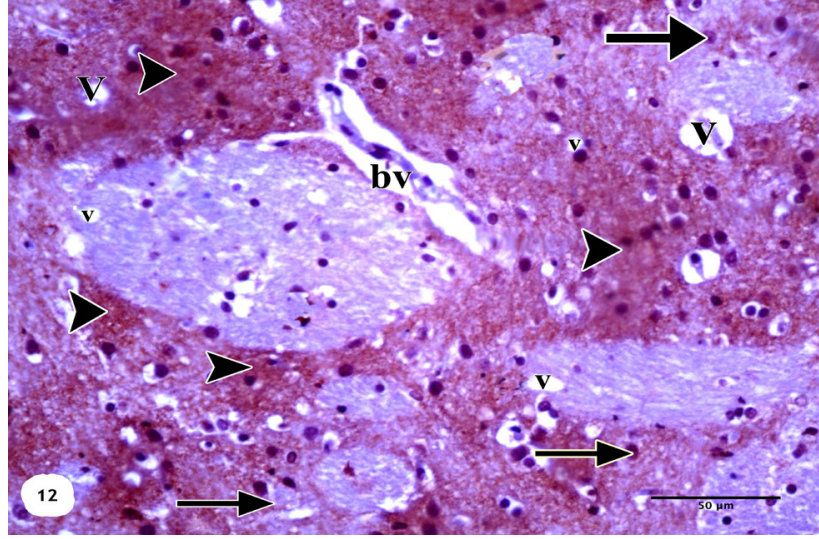

Fig. 12: A photomicrograph of adult treated rat putamen showing some areas with weak positive TH immunoreactivity (arrow heads) and other areas with a negative TH immunoreactivity in the neuopil and neurons (arrow). The dilated blood vessel (bv) is noticed. The striatal fibers and neuropil show many vacuoles (v). $\quad \mathrm{TH}$, counterstained with $\mathrm{H}$., $\times 400$

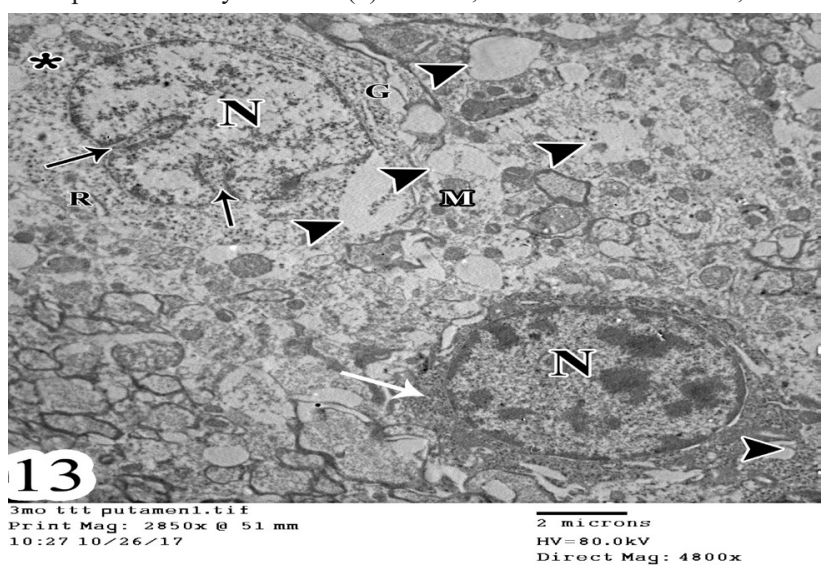

Fig. 13: An electron micrograph of an ultrathin section in the putamen of adult treated rat showing two neurons. One neuron has a heterochromatic nucleus $(\mathrm{N})$ and the cytoplasm appears electron dense (white arrow) with vacuolations (arrow heads) and a marked loss of cell organelles. Another neuron has an irregular nucleus (N) with deep invaginations of the nuclear envelope (arrows). The cytoplasm shows dilated rough endoplasmic reticulum (R), dilated Golgi apparatus $(\mathrm{G})$, vacuolations (arrowheads), damaged mitochondria $(\mathrm{M})$ and some polyribosomes (asterisk)

TEM, $\times 4800$

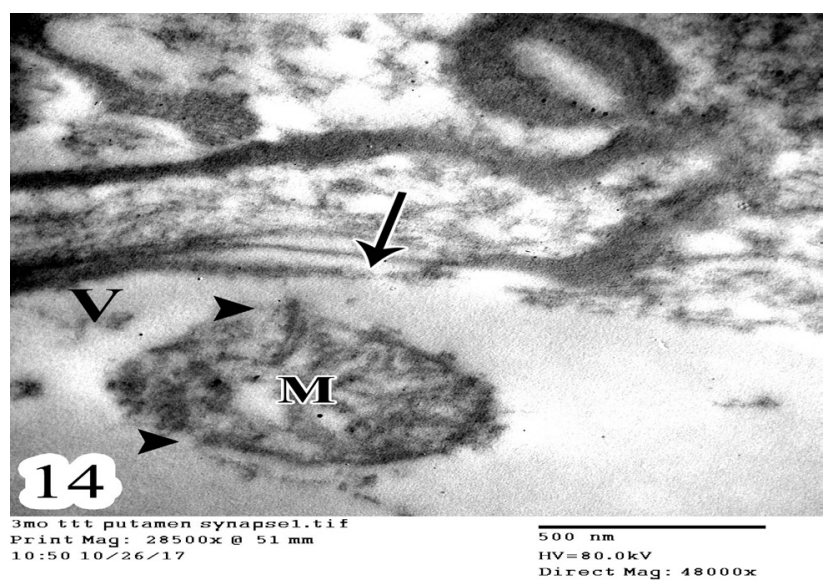

Fig. 14: An electron micrograph of an ultrathin section in the putamen of adult treated rat showing synapse with an area of loss of presynaptic and postsynaptic densities (arrow) and minute number of the synaptic vesicles (V). Swollen mitochondria (M) with destructed inner cristae are observed and its wall is broken at several points (arrow heads). TEM, $\times 48000$ 


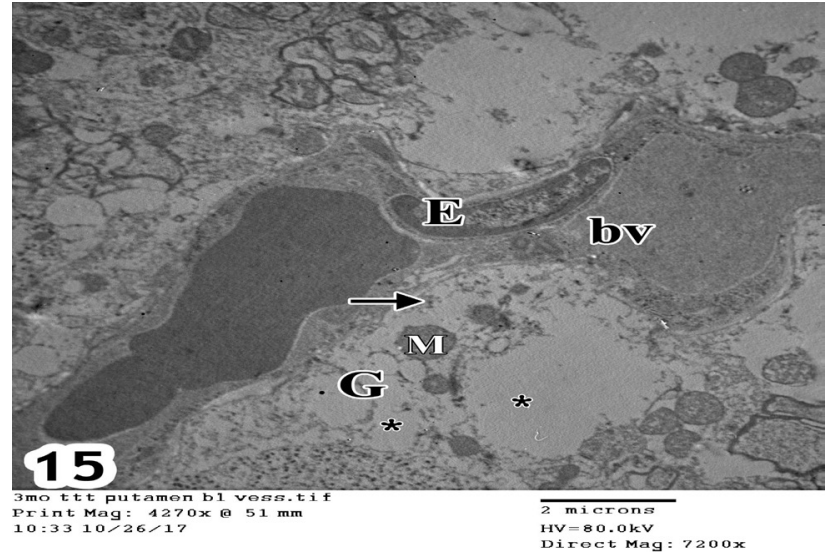

Fig. 15: An electron micrograph of an ultrathin section in the putamen of adult treated rat showing the blood vessel (bv). The endothelial cell (E) has a dark nucleus with coarse clumps of chromatin. The pericytic microglia (G) show big vacuolations (asterisks), damaged mitochondria (M) and rarified cytoplasm (arrow). TEM, $\times 7200$

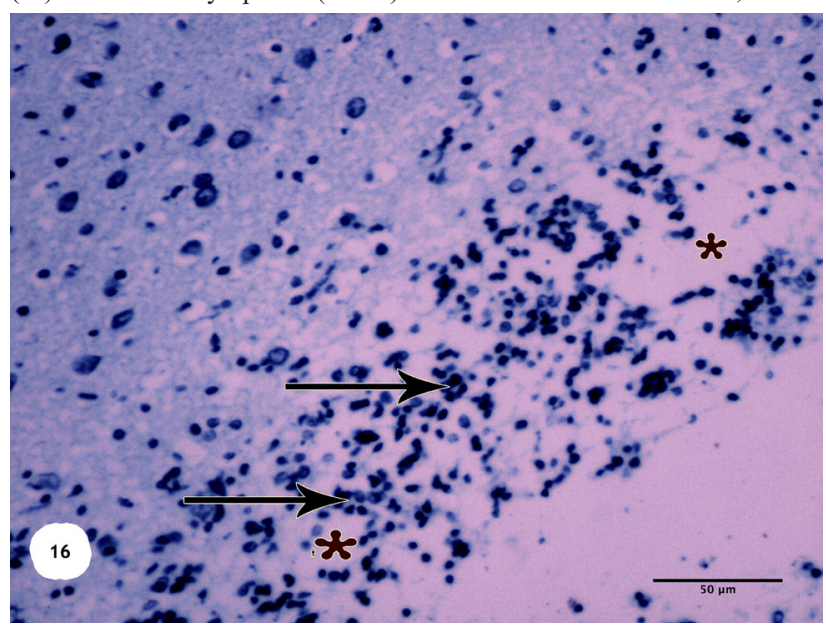

Fig. 16: A photomicrograph of the subthalamic nucleus of adult treated rat showing wide spaces (asterisks) between darkly stained neurons (arrows).

Gallocyanine, $\times 400$

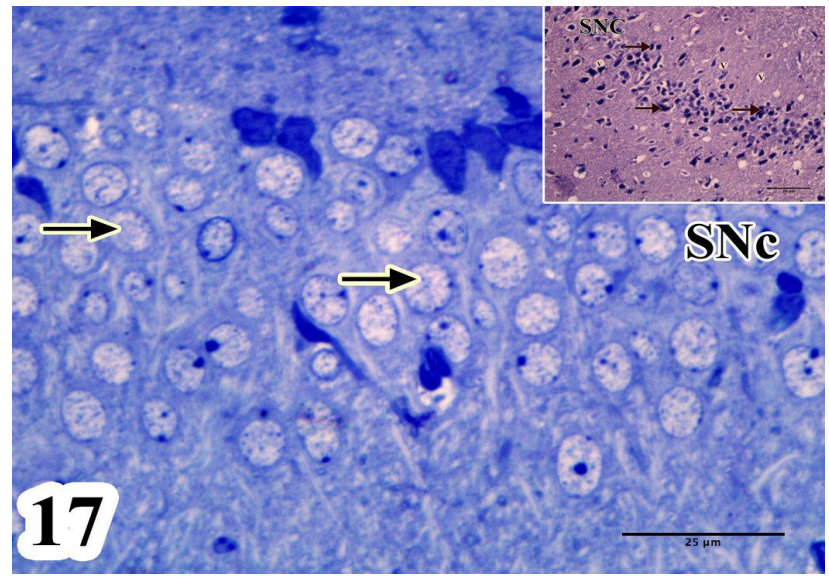

Fig. 17: A photomicrograph of a semithin section in the substantia nigra pars compacta $(\mathrm{SNc})$ of adult treated rat showing apparently increased interstitial spaces between neurons and a reduction number of neuronal perikarya in comparison with the control. The neurons appeared normal (arrow). Inset: A photomicrograph of adult treated rat midbrain in the substantia nigra pars compacta $(\mathrm{SNc})$ showing varying sizes and shapes of the neuronal perikarya. The neuropil shows many vacuoles (V). Most of the neurons (arrows) appear to have darkly stained nuclei. Toluidine blue, $\times 1000$ and Inset: Gallocyanine, $\times 400$

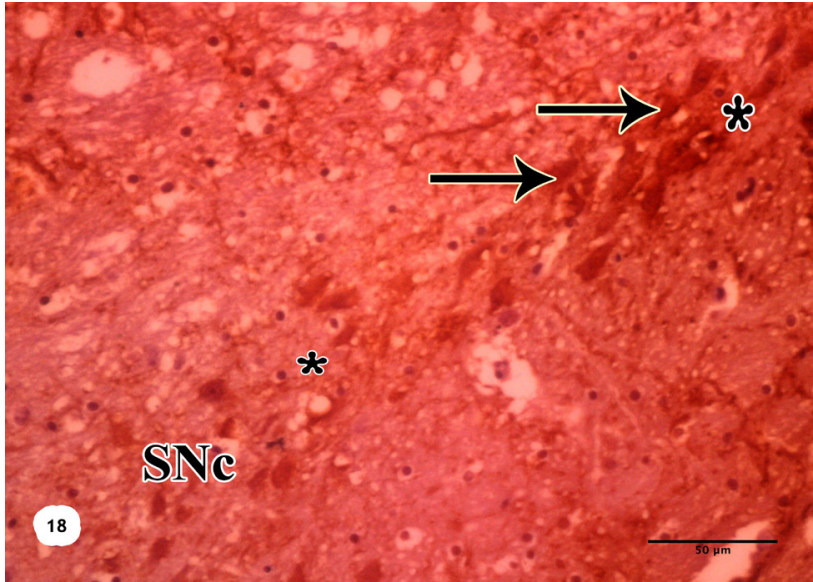

Fig. 18: A photomicrograph of adult treated rat substantia nigra par compacta $(\mathrm{SNc})$ showing weak $\mathrm{TH}$ immunoreactivity and decreased in number of TH immunopositive neurons (arrow) and mild immunoreactive neuropil (asterisks). $\quad \mathrm{TH}$, counterstained with $\mathrm{H}, \times 400$

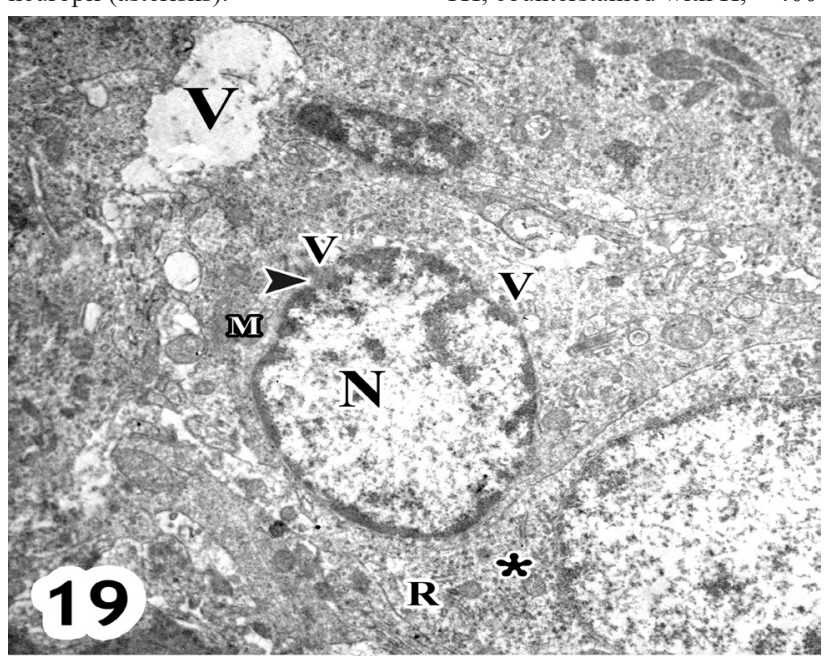

Fig. 19: An electron micrograph of an ultrathin section in the substantia nigra pars compacta of adult treated rat showing a shrunken neuron with a part of another neuron. The nucleus $(\mathrm{N})$ shows peripheral condensation of the chromatin with irregular outline and a point of breakage in the nuclear membrane (arrow head).The cytoplasm shows damaged mitochondria $(\mathrm{M})$, dilated rough endoplasmic reticulum (R), vacuoles $(\mathrm{V})$ and free polyribosomes (asterisk).

TEM, $\times 7200$

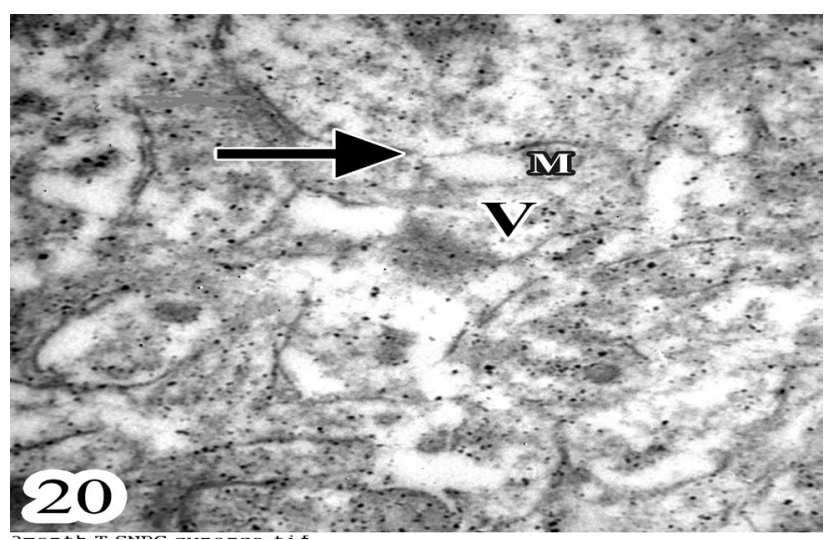

Fig. 20: An electron micrograph of an ultrathin section in the substantia nigra pars compacta of adult treated rat showing synapse with an area of loss of presynaptic and postsynaptic densities (arrow), a few synaptic vesicles $(\mathrm{V})$ and destructed mitochondria with breakage of its inner cristae (M). 
Table 1: The number of cells in the putamen per an area of $313.4 \mu^{2}$ in the control and treated groups

\begin{tabular}{lccccc}
\hline & Mean & $\mathrm{N}$ & $\begin{array}{c}\text { Std. } \\
\text { Deviation }\end{array}$ & $\begin{array}{c}\text { Std. Error of } \\
\text { the Mean }\end{array}$ & p-value \\
\hline Control & 51.6 & 20 & 2.2 & 0.5 & $0.000^{*}$ \\
Treated & 31.0 & 20 & 2.9 & 0.6 & \\
\hline
\end{tabular}

*statistical significant difference $(\mathrm{P}<0.001)$

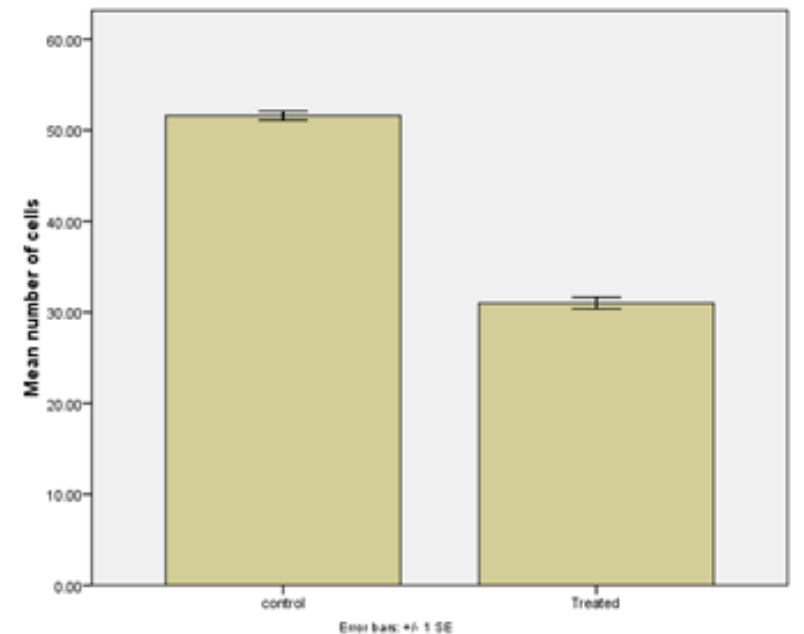

Histogram (1): showing the number of cells of putamen per an area of $313.4 \mu^{2}$ in the control and treated groups

Table 2: The surface area of neurons in the putamen per an area of $124.2 \mu^{2}$ in the control and treated groups

\begin{tabular}{lccccc}
\hline & Mean & $\mathrm{N}$ & $\begin{array}{c}\text { Std. } \\
\text { Deviation }\end{array}$ & $\begin{array}{c}\text { Std. Error of } \\
\text { the Mean }\end{array}$ & p-value \\
\hline Control & 100.4 & 20 & 2.5 & 0.5 & $0.000^{*}$ \\
Treated & 64.2 & 20 & 4.9 & 1.1 & \\
\hline
\end{tabular}

*statistical significant difference $(\mathrm{P}<0.001)$

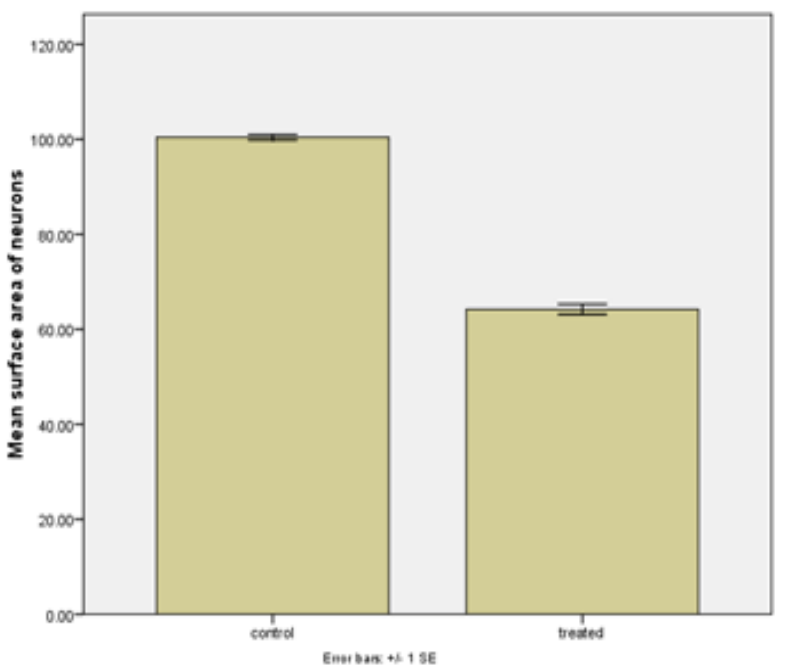

Histogram (2): showing the surface area of neurons in the putamen per an area of $124.2 \mu^{2}$ in the control and treated groups
Table 3: The number of cells in SNc per an area of $313.4 \mu^{2}$ in the control and treated groups

\begin{tabular}{lccccc}
\hline & Mean & $\mathrm{N}$ & $\begin{array}{c}\text { Std. } \\
\text { Deviation }\end{array}$ & $\begin{array}{c}\text { Std. Error of } \\
\text { the Mean }\end{array}$ & p-value \\
\hline Control & 62.2 & 20 & 1.3 & 0.2 & $0.000^{*}$ \\
Treated & 29.1 & 20 & 1.4 & 0.3 & \\
\hline
\end{tabular}

*statistical significant difference $(\mathrm{P}<0.001)$

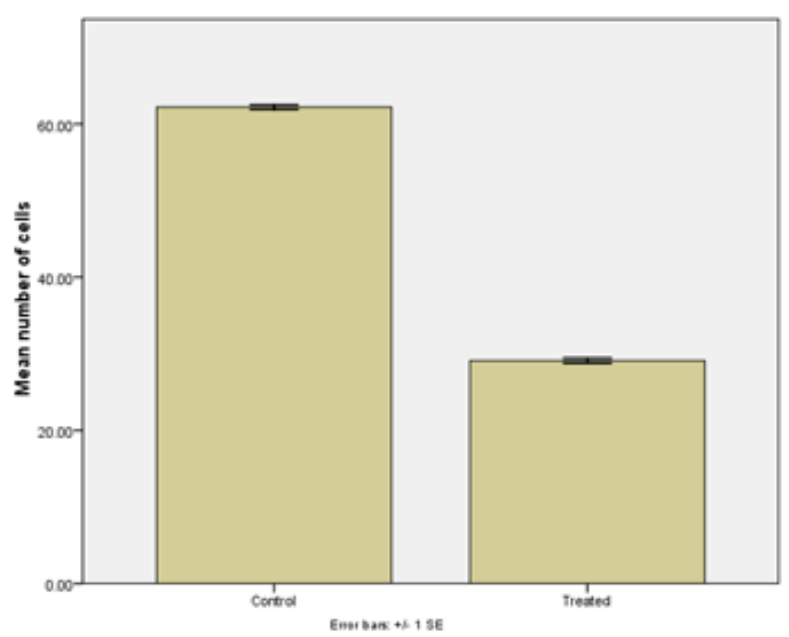

Histogram (3): showing the number of cells of SNc per an area of 313.4 $\mu^{2}$ in the control and treated groups

Table 4: The surface area of neurons in the SNc per an area of $124.2 \mu^{2}$ in the control and treated groups

\begin{tabular}{lccccc}
\hline & Mean & $\mathrm{N}$ & $\begin{array}{c}\text { Std. } \\
\text { Deviation }\end{array}$ & $\begin{array}{c}\text { Std. Error of } \\
\text { the Mean }\end{array}$ & p-value \\
\hline Control & 125.0 & 20 & 1.7 & 0.3 & $0.000^{*}$ \\
Treated & 25.1 & 20 & 3.4 & 0.7 & \\
\hline
\end{tabular}

*statistical significant difference $(\mathrm{P}<0.001)$

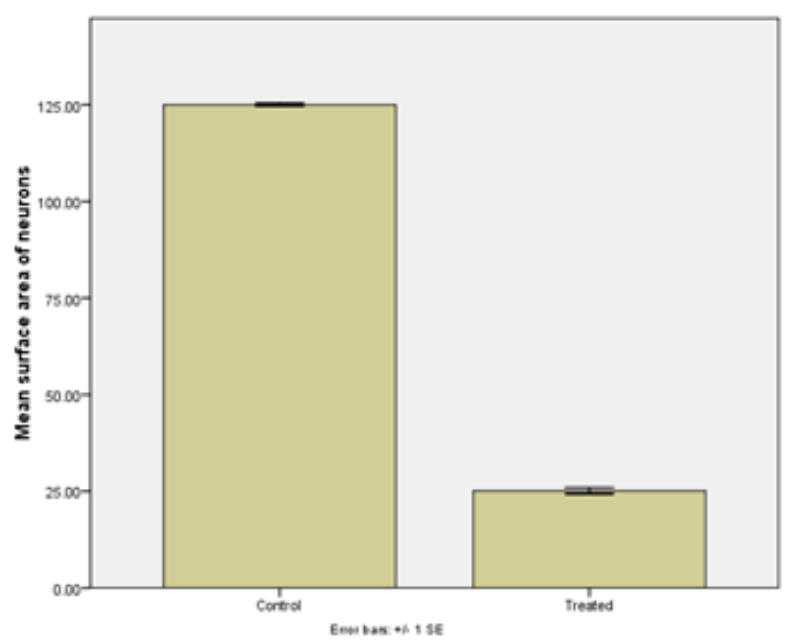

Histogram (4): showing the surface area of neurons in SNc per an area of $124.2 \mu^{2}$ in the control and treated groups 


\section{DISCUSSION:}

It has been reported that iron transport and transferrin binding sites which responsible of cerebral iron uptake are maximal during the postnatal period since rapid brain growth, especially during the second week postnatal in rats and mice. So iron needed by the brain during this period is retained in the brain without being returned to plasma sites. This fact clarifies that the effect of iron dose is augmented if demonstrated during postnatal period ${ }^{[1]}$.

\section{The putamen}

The present study revealed that neurons in the control group had vesicular nuclei and a basophilic cytoplasm due to Nissl's granules. The neurons were surrounded by perineuronal spaces since myelin did not pick up the stain; the myelination was formed by oligodentrocytes. The putman consists largely of medium- to small-sized neurons with spiny dendrites (medium spiny neurons), which use gamma-aminobutyric acid (GABA) as their neurotransmitter ${ }^{[17]}$.

The putamen in the treated group of this study showed neurons that had dense darkly stained nuclei and some neurons with vesicular nuclei. The neurons are surrounded by perineuronal spaces. The blood vessels appeared dilated. Some vacuolations appeared within the neuropil. These findings were in the same line with previous study ${ }^{[5]}$.

The intake of high iron in the diet was reported to cause iron accumulation in the brain and produces significant cognitive deficits as impairments in spatial memory, aversive memory, and recognition memory in rodents and it is a risk factor for Parkinson's disease ${ }^{[8,12,18-20]}$.

Immunohistochemistry of the putamen in the control group revealed many positive $\mathrm{TH}$-immunoreactive neurons and a positive $\mathrm{TH}$ immunoreactivity in the striatal fibers and neuropil. This is in accordance with previous study that reported that dopamine innervation of the striatum is relatively dense and uniform and this related to the underlying organization of the nigrostriatal system into patch and matrix-directed systems ${ }^{[21]}$.

On the other hand, immunohistochemistry of the putamen in the treated group using anti- $\mathrm{TH}$ demonstrated some areas with a positive $\mathrm{TH}$ immunoreactivity with relative reduction in the intensity of immunoreativity and other areas with a negative TH immuno reactivity. These changes were in agreement with previous studies on the adult mice fed with $\mathrm{Fe}$ during the neonatal period that showed reduced striatal dopamine content ${ }^{[22,23]}$. The present results were explained by study that concluded that the reduction in striatum dopamine content due to iron induced nigral degeneration. The iron catalyzes the amines by monoamine oxidase with highly toxic free radicals released through the Fenton reaction ${ }^{[1]}$.

The neurons of the putamen of the control group had an euchromatic nucleus with a prominent nucleoli. The cytoplasm showed Golgi bodies, rough endoplasmic reticulum, many free polyribosomes and many mitochondria.

Examination of the symmetrical synapses in the control group, many synaptic vesicles and mitochondria and blood vessels are seen with normal endothelial cells and pericytic microglia. That is in agreement with studies on the ultrastructure of the dopaminergic terminals in the striaturn ${ }^{[24,25,26,27]}$. The examined the synapses within the striatum and detected that the $\mathrm{TH}+$ terminals and the $\mathrm{DA}+$ terminals were rich in vesicles, and some contained mitochondria. The vesicles within both the $\mathrm{TH}+$ and the $\mathrm{DA}+$ terminals were predominantly small to medium-sized, but a small number of large vesicles were also observed ${ }^{[21]}$.

In contrast, there was an apparent loss of the cell organelles and a damaged nucleus of neurons in the putamen of treated group in this study. This is in agreement with other researches ${ }^{[1,}{ }^{19]}$. The hydroxyl radical damage to DNA and RNA probably led to cellular necrosis might explain the present changes. Iron caused a decreased AChE activity in the striatum when compared to controls ${ }^{[19]}$. The results suggest that, iron caused cognitive deficits are related to impairment of cholinergic neural transmission in the brain. These findings might have implications for the development of new therapeutic strategies aimed at improving cognitive decline observing in the neurodegenerative disorders.

At the site of synaptic contact, in this work an area of loss of presynaptic and postsynaptic densities and the synaptic terminal showed minute number of the synaptic vesicles. Swollen mitochondria with destructed inner cristae were observed and its wall was broken at several points. This might also be a consequence of iron administration that increased superoxide production in submitochondrial particles, suggesting mitochondrial dysfunction and mitochondria have many death factors that are released upon apoptotic stimuli[ ${ }^{[27]}$.

\section{The subthalamic nucleus}

The present work demonstrated that the subthalamic nucleus in the control group consists of closely packed dense neurons forming characteristic biconvex shape. The neuron of subthalamic nucleus had a variance in the diameters of body of the cell and dendritic ramifications ${ }^{[28]}$.

The subthalamic nucleus in treated group showed wide spaces between darkly stained neurons. The changes could be attributed to striatal dopamine depletion, the hallmark of Parkinson's disease, which associated with an abnormal activity of the subthalamic nucleus ${ }^{[29]}$. This was supported by the study that declared that discrete dopamine depletion produced by infusing the neurotoxin 6-hydroxydopamine (6-OHDA) bilaterally into the dorsal part of the striatum, produced defects in the initiation of motor activity which were revealed by an increment in the number of delayed responses and a lengthening of the reaction times (RTs) of the onset of electromyographic activity during initiation of a contraction of the muscles which indicated the STN 
nucleus lesion ${ }^{[30]}$. The previous study also supported that the concept of a predominant control of the STN on basal ganglia output structures ${ }^{[30]}$.

TH immunostaining of the subthalamic nucleus in this work had not been performed because the neurotransmitter in the subthalamic nucleus is glutamate and anti- TH is not specific. The semithin and ultrathin sections of the subthalamic nucleus were technically difficult.

\section{The substantia nigra pars compacta}

$\mathrm{SNc}$ in the control group of this study appeared as a collection of closely packed neurons that had variable sizes and shapes of neuronal perikarya. Their cytoplasm showed moderate to intense basophilia. These findings were in line with the study that declared that the dimensions of SNc neurons were on average larger than SN pars reticulate ${ }^{[31]}$. The present results were in accordance with previous study that added that the neurons appeared multipolar or bipolar, with infrequently branching dendrites ${ }^{[32]}$. Dendrites appeared with finely scattered spines and dendritic varicosities. Spines could also be seen on the cell soma.

Immunohistochemistry of $\mathrm{SNc}$ in the control group showed many positive TH-immunoreactive neurons. The present results agreed with the study that described dopaminerigic neurons of $\mathrm{SNc}$ labeled with tyrosine hydroxylase immunoreactivity as densely packed but the pars reticulata had relatively sparse cells compared to the pars compacta ${ }^{[33]}$.

The dense positive TH-immunoreactive neuropil was seen in the SNc of the control group. This could be explained by the study that reported that the neurons of SNc possess dendrites that extend into the pars reticulata that gives rise a positive TH immunoreaction in the neuropil ${ }^{[34]}$.

In the present work, $\mathrm{TH}$ immunostaining of $\mathrm{SNc}$ in the experimental group showed a negative $\mathrm{TH}$ immunoreactivity and reduction in the number of $\mathrm{TH}$ immunopositive neurons and TH immunoreactive neuropil in comparison with the control group. These findings were in agreement, an iron dextran overload led to an increase in the iron content in the $\mathrm{SN}$, a decreased dopamine release and content, and a reduced the numbers of $\mathrm{TH}$ immunoreactive neurons ${ }^{[35]}$.

On the other hand, the mice was injected with an iron overdose did not display obvious motoric deficits with the age, suggesting that iron accumulation in the $\mathrm{SN}$ alone is insufficient to promote neurodegeneration ${ }^{[36]}$.

In this study, the cell bodies of the neurons in the $\mathrm{SNc}$ in the control group showed a large euchromatic nucleus with a prominent nucleolus. The cytoplasm contained distinct aggregates of short RER cisternae with many free polyribosomes between them and scattered mitochondria. The present results were in agreement with other studies ${ }^{[32,37]}$. Asymmetrical synapses with many synaptic vesicles and mitochondria were observed by electron examination of the SNc in this study. These were in accordance the structure of synapse in mammalian brain. The active zone is a particular region on the presynaptic plasma membrane, where synaptic vesicles are anchored and primed for release ${ }^{[38]}$.

The morphological alterations were observed in both the nucleus and the cytoplasm in the treated group. Some shrunken pyknotic neurons were seen. The nuclei showed condensation of the chromatin in the periphery with irregular outline and points of breakage in the nuclear membrane. The cytoplasm showed few damaged mitochondria, dilated rough endoplasmic retinaulum and vacuoles. The present finding, commensurate with the result of previous study that reported the intranigral infusion of iron alone to adult rats produced dose-dependent neuronal death of $\mathrm{SNc}^{[39]}$. The present nuclear changes could be explained by the damage to DNA and RNA that was induced by the iron overdose due to the oxidative stress ${ }^{[5]}$. This is also in agreement with the study that reported that iron administration caused decreased number of dopaminergic neurons of $\mathrm{SNc}^{[8]}$.

The degenerative changes of putamen, subthalamic nucleus and $\mathrm{SNc}$ in the present study might be attributed to Fe complexes that generate reactive oxygen species (ROS) such as hydrogen peroxide $\left(\mathrm{H}_{2} \mathrm{O}_{2}\right)$ and nitric oxide which has been implicated in neuronal toxicity because the oxidation of lipids, proteins, polysaccharides and DNA damage (apoptosis) ${ }^{[12,40,41,42,43,44]}$. The other cause of present degeneration might to be that iron stimulate the oxidation of dopamine and facilitate the formation of dopamine quinone as well as the neurotoxic 6-OHDA ${ }^{[45]}$.

Moreover, the increment concentrations of iron in the brain contribute to neurodegenerative processes has also been accepted. Neuroferritinopathy is a dominantly inherited, late-onset disease of the basal ganglia that presents with extrapyramidal features similar to those of Huntington's and Parkinson's diseases. Patients with neuroferritinopathy have abnormal accumulation of iron and ferritin in the brain and low ferritin concentrations in the blood lead to a mutation in the gene for ferritin light polypeptide. Iron can also enhance the aggregation of a-synuclein, which is particularly toxic to DA neurons ${ }^{[46]}$.

Nonetheless, the present results suggest that chronic iron overdose cause destruction of the striatum and substantia nigra. A better understanding of the functional consequences of the abnormality in iron regulation in aging and neurological diseases may identify new targets for treating memory defects that affect aging population which will be a major sector in the society. So, further studies are required to test if the dietary iron restriction is beneficial in Parkinson's disease or not.

\section{REFERENCES:}

1. Matta. A., Henrique, C., Farinhas, J., Masiero, L., Teixeira, S., Marinho, V., Bastos, V., Pedro, R. and de Medeiros, L.2017. Iron Accumulation and 
Neurodegeneration in Patients with Alzheimer's Diseases: An Integrative Review Study of the Evidence". EC Neurology 6: 267-272.

2. Hoepken, H.H., Korten, T., Robinson, S.R. and Dringen, R. 2004. Iron accumulation, iron mediated toxicity and altered levels of ferritin and transferrin receptor in cultured astrocytes during incubation with ferric ammonium citrate. Journal of Neurochemistry 88: 1194-1202.

3. Thompson, K.J., Shoham, I.J. and Connor, J.R. 2001. Iron and neurodegenerative disorders. Brain Research Bulletin 55: 155-164.

4. Weinreb, O., Mandel, S., Moussa, B., Youdim, H. and Amit, T. 2013. Targeting dysregulation of brain iron homeostasis in Parkinson's disease by iron chelators. Free Radical Biology and Medicine 41:55-58.

5. Jeanelle, Ariza, S., Hailee, Rogers B.S., Anna, H. B., Melissa, Snell, B.S., Michael, D. B. and Derek, Judd B.S. 2017. Iron accumulation and dysregulation in the putamen in fragile $\mathrm{X}$-associated tremor/ataxia syndrome. Movement disorders 32(4):585-591.

6. Zecca, L., Gallorini, M., Schunemann,V. ,Trautwein, A.X. , Gerlach, M. , Riederer, P. , Vezzoni, P. and Tampellini, D. 2001. Iron, neuromelanin and ferritin content in the substantia nigra of normal subjects at different ages: consequences for iron storage and neurodegenerative processes. Journal of Neurochemistry 76: 1766-1773.

7. Fedorow, H., Tribl, F., Halliday, G., Gerlach, M. , Riederer, P. and Double, K.L. 2005. Neuromelanin in human dopamine neurons: comparison with peripheral melanins and relevance to Parkinson's disease. Progress in Neurobiology 75:109-124.

8. Carboni, E., Tatenhorst, L., Tönges, L., Barski, E., Dambeck, V., Bähr, M and Lingor, P. 2017. Deferiprone rescues behavioral deficits induced by mild iron exposure in a mouse model of alphasynuclein aggregation. NeuroMolecular Medicine 19(2):309-321.

9. Schipper, H.M. 2012. Neurodegeneration with brain iron accumulation clinical syndromes and neuroimaging. Biochimica et Biophysica Acta $822: 350-360$

10. Yoshiyuki, T., Setsuko, T., Cho, A., Takeshi, M., Lining, K. and Nutcharin, O. 2013. Mineral composition of and the relationships between them of human basal ganglia in very old age. Life Sciences 151:18-29.

11. Dal-Pizzol, F.,Klamt, F., Frota, M.L., Andrades,M.E., Caregnato, F.F. , Vianna, M., Schröder, N., Quevedo, J. , Izquierdo, I. , Archer, T. and Moreira, J.C. 2001. Neonatal iron exposure induces oxidative stress in adult Wistar rat. Developmental brain research 23(1):109-114.
12. De Lima, M.N., Polydoro, M. , Laranja, D.C. , Bonatto, F. , Bromberg, E. , Moreira, J.C. , Dal-Pizzol, F. and Schröder, N. 2005. Recognition memory impairment and brain oxidative stress induced by postnatal iron administration. European Journal of Neuroscience 21(9):2521-2528.

13. Bancroft, J.D. and Gamble, M. 2008. Theory and practice of histological techniques. 6th ed.Churchill Livingstone / Elsevier, Philadelphia, PA, Edinburgh.

14. Hwang, D., Ardayfio, P., Jung, K., Elena, V., Semina, and Kwang-Soo, K. 2003. Selective loss of dopaminergic neurons in the substantia nigra of Pitx3deficient aphakia mice. Molecular Brain Research, 114: 123-131.

15. Kue, J. 2007. Electron microscopy methods and protocols. New Jersey. Springer. Science and Business Media CH: 3, PP: 1-8.

16. Dawson, B.and Trapp, R.G. 2001. Basic and clinical biostatistics. 3rd ed. New York: Lange Medial books/ Mc Graw-Hill.

17. Lanciego, J. L., Luquin, N. and Obeso, J. A. 2012. Functional Neuroanatomy of the Basal Ganglia. Cold Spring Harbor Perspectives in Medicine 2(12): 9621.

18. Johnson, C.C., Gorell, J.M., Rybicki, B.A., Sanders, K. and Peterson, E.L. 1999. Adult nutrient intake as a risk factor for Parkinson's disease. International Journal of Epidemiology 28:1102-1109.

19. Perez, V.P. De Lima M.N. , Da Silva, R.S. Dornelles, A.S. , Vedana, G. , Bogo, M.R., Bonan, C.D. and Schröder, N. 2010. Iron leads to memory impairment that is associated with a decrease in acetylcholinesterase pathways. Current Neurovascular Research 7(1):15-22.

20. Schröder, N., Figueiredo, S.L. and de Lima, M.N. 2012. Role of Brain Iron Accumulation in Cognitive Dysfunction: Evidence from Animal Models and Human Studies. The Journal of Alzheimers Diseases 34(4):797-812.

21. Ellen, J., Karela, K., Anderson, L. M. and Anton, R. 1996. Light and electron microscopic immunohistochemical study of dopaminergic terminals in the striatal portion of the pigeon basal ganglia using ant isera against tyrosine hydroxylase and dopamine. The journal of comparative neurology 369:109-124.

22. Fredriksson, T. and Archer, A. 2003. Effect of postnatal iron administration on MPTP- induced behavioral deficits and neurotoxicity: behavioral enhancement by L-Dopa-MK-801 co-administration. Behavioural brain research139: 31-46.

23. Bueno-Nava, A. , Gonzalez-Pina, and RalfaroRodriguez, A. 2010. Iron-dextran injection into the 
substantia nigra in rats decreases striatal dopamine content ipsilateral to the injury site and impairs motor function. Metabolic Brain Diseases 25(2):235-9.

24. Triarhou, L.C., Norton, J. and Ghetti, B. 1988. Synaptic connectivity of tyrosine hydroxylase immunoreactive nerve terminals in the striatum of normal, heterozygous and homozygous weaver mutant mice. Journal of neurocytology 17:221-232.

25. Aoki, C. and Pickel, V. M. 1988. Neuropeptide Y-containing neurons in the rat striatum: ultrastructure and cellular relations with tyrosine hydroxylasecontaining terminals and with astrocytes. Brain Research. 459, 205-225.

26. Ovtscharoff, W. 1992. Gamma-aminobutyric acid, glutamic acid decarboxylase and tyrosine in rat striatum demonstrated by single and dual immunocytochemistry. The Journal of comparative neurology 33:311-319.

27. Taylor, D.L., Edwards, A.D. and Mehmet, H. 1999. Oxidative metabolism, apoptosis and perinatal brain injury. Brain Pathology 9(1):93-117.

28. Kita, H., Chang, H. T. and Kitai, S. T. 1983. The morphology of intracellularly labeled rat subthalamic neurons: A light microscopic analysis. Journal of Comparative Neurology 215: 245-257.

29. Darbaky,Y., Claude, F., Marianne, A. and Christelle, B.2003. High frequency stimulation of the subthalamic nucleus has beneficial antiparkinsonian effects on motor functions in rats, but less efficiency in a choice reaction time task. European Journal of Neuroscience 18(4):951-956

30. Baunez, C., Nieoullon, A. A and Amalric, M. 1995. In a rat model of parkinsonism, lesions of the subthalamic nucleus reverse increases of reaction time but induce a dramatic premature responding deficit. Journal of Neuroscience 15 (10):6531-6541.

31. Yelnik, J., Francois, C., Percheron, G. and Heyner, S. 1987. Golgi study of the primate substantia nigra. I. Quantitative morphology and typology of nigral neurons. Journal of Comparative Neurology 265, 455-472.

32. Abdel-Hafez, A.M. and Mohamed, H. K. 2013. Sex differences in dopaminergic neurons of substantia nigra pars compacta of adult and aged rats: a histological and immunohistochemical study.The Egyptian Journal of Histology 36:33-42.

33. Depaepe V, Cuvelier L, Thöny B, Résibois A.Pterin4alpha-carbinolamine dehydratase in rat brain. I. Patterns of co-localization with tyrosine hydroxylase. Brain Res Mol Brain Res. 2000;75(1):76-88.
34. Tepper, J. M., Sawyer, S. F. and Groves, P. M. 1987. Electrophysiologically identified nigral dopaminergic neurons intracellularly labeled with HRP: Lightmicroscopic analysis. Journal of Neuroscience 7, 2794-2806.

35. Hong, J., Zhuo, L., Wang, J. and Xie, J. 2006. Neuroprotective effects of iron chelator Desferal on dopaminergic neurons in the substantia nigra of rats with iron-overload. Neurochemistry International 49: 605-609.

36. Cheng-Wu, Z. Yee, Kit, T. , Bing-Han, C. , Katherine, C. M. , Eng-Tat ,A., Fai, T., Bryce, W. Q., Eugenia, T. E., Abu Bakar, A.A., Kai-Hsiang, C. , Kah-Leong, L. and Tuck, W.2017. Transgenic Mice Overexpressing the Divalent Metal Transporter 1 Exhibit Iron Accumulation and Enhanced Parkin Expression in the Brain. NeuroMolecular Medicine 19:375-386.

37. Sailaja, K. and Gopinath, G. 1996. Ultrastructure of developing substantia nigra in humans. The International Journal of Developmental Neuroscience 14:761-770.

38. Harris, K.M. and Weinberg, R.J.2012. Ultrastructure of synapses in the mammalian brain. Cold Spring Harbor Perspectives in Medicine 5:1-4.

39. Sengstock, G.I. , Olanow, C.W. , Menzies, R.A. , Dunn, A.J. and Arendash, G.W.1993. Infusion of iron in the rat substantia nigra: nigral pathology and dosedependent loss of striatal dopaminergic markers. The Journal of Neuroscience Research 35:67-82.

40. Powers, K.M. Smith-Weller, T. Franklin, G.M. Longstreth, W.T. Swanson, P.D. and Checkoway, H. 2003. Parkinson's disease risks associated with dietary iron, manganese, and other nutrient intakes. Neurology 60: 1761-1766.

41. Ben-Shachar, D., Kahana, N., Kampel, V., Warshawsky, A. and Youdim, M.B. 2004. Neuroprotection by a novel brain permeable iron chelator, VK-28, against 6-hydroxydopamine lession in rats. Neuropharmacology 46 (2): 254-263.

42. Zecca, L., Youdim, M.B., Riederer, P., Connor, J.R. and Crichton, R.R. 2004. Iron, brain ageing and neurodegenerative disorders. Nature Reviews Neuroscience 5:863-873.

43. Youdim, M.B., Fridkin, M. and Zheng, H. 2005. Bifunctional drug derivatives of MAO-B inhibitor rasagiline and iron chelator $\mathrm{VK}-28$ as a more effective approach to treatment of brain ageing and ageing neurodegenerative diseases. Mechanisms of Ageing and Development 126: 317-326.

44. Barnham, K. J. and Bush, A. I. 2008. Metals in 
Alzheimer's and Parkinson's Diseases. Chemical Biology 12:222-228.

45. Hare, D. J. and Double, K. L. 2016. Iron and dopamine: A toxic couple. Brain. 139:1026-1035.
46. Levin, J., Hogen, T., Hillmer, A. S., Bader, B. Schmidt, F. and Kamp, F. 2011. Generation of ferric iron links oxidative stress to alpha-synuclein oligomer formation. Journal of Parkinsons Diseases 1(2), 205-216. 
الملخص العربى

\section{دراسة نسيجية وكيمياء نسيجية مناعية على تاثير جرعة الحديد الزائدة على الانوية

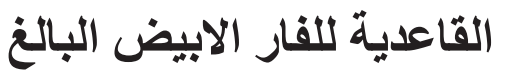

\section{محمد نبيل محمود صالح، محمد البدرى محمد، ايمن صلاح الدين عامر، امنية ابراهيم اسماعيل

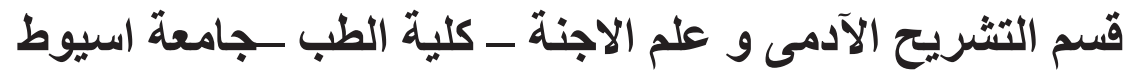

المقدمة: يعتبر الحديد من اكثر العناصر المنتشرة على الارض و هو عنصر الكاسي للحياة لانه بيتخدم بكثرة فى نركيب

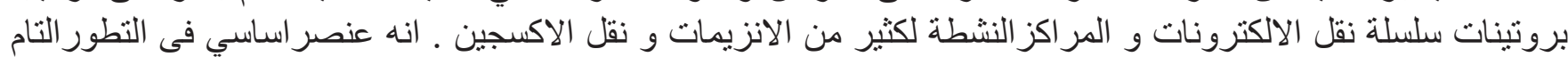

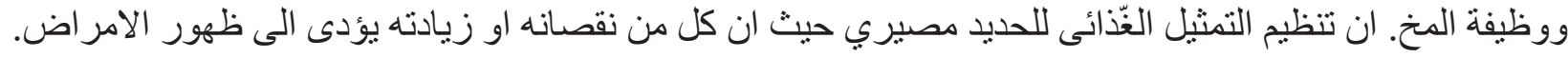

الههف من البحث: تحديد تاثثير تعرض الحديد الزائد اثناء فترة بعد الو لادة على البيوتامين والنواة تحت المهاد و الجزء المكتنز

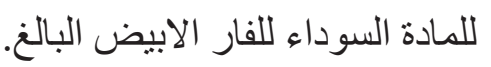

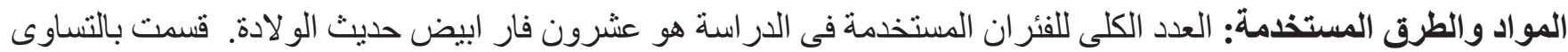

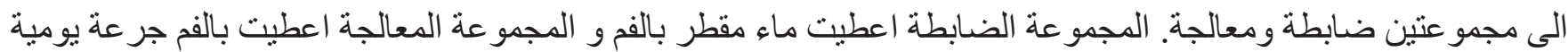

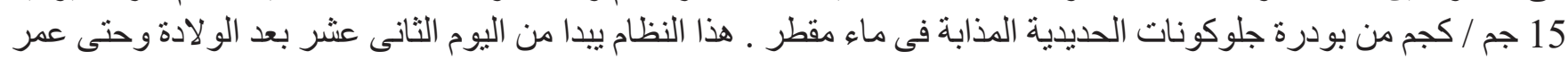

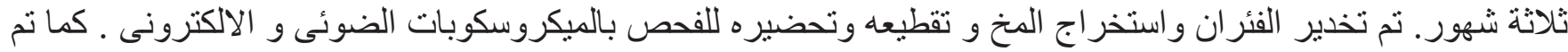
اجر اء در اسة مورفومترية.

النتائج: اوضحت الدراسة بالميكروسكوب الضونئ للمجموعة المعالجة بالحديد ان الخلايا العصبية للبيوتامين اصبحت النيات انويتها

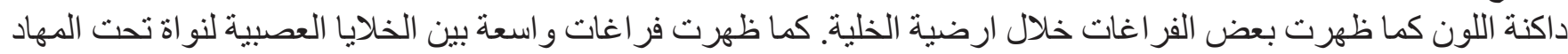

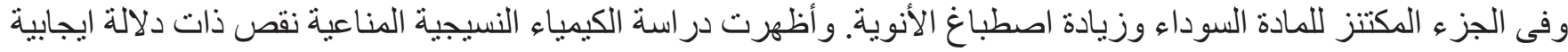

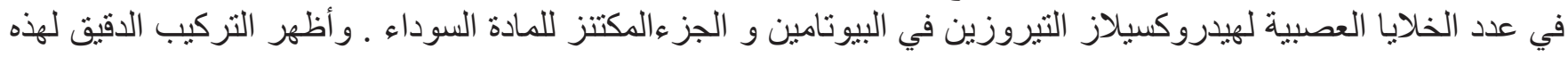

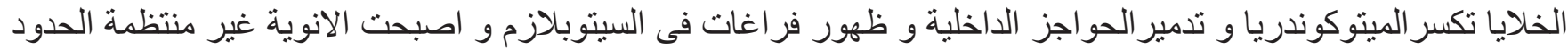

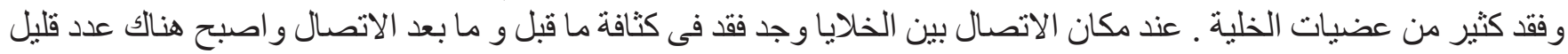

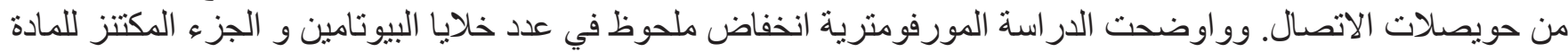

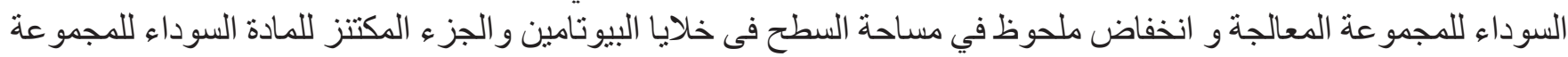
المعالجة.

الخلاصة: تتاول جر عة الحديد الز ائدة اثناء فترة ما بعد الو لادة ادت الى تغير ات تتكسية فى البيوتامين و النواة تحت المهادو الجزء

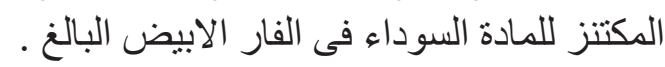

